\title{
The influence of phonon anharmonicity on thermal and elastic properties of neptunium
}

\author{
A. Filanovich*, A. Povzner \\ Ural Federal University, Mira Str. 19, 620002 Ekaterinburg, Russia
}

\section{A R T I C L E I N F O}

\section{Article history:}

Received 2 September 2012

Accepted 27 January 2013

Available online 8 February 2013

\begin{abstract}
A B S T R A C T
A self-consistent thermodynamic model describing the thermal and elastic properties of $\alpha$-and $\beta$-phases of neptunium was developed. The presence of strong phonon anharmonicity of $\mathrm{Np}$ is established. The obtained results are in good agreement with the experimental data and enable to predict the Np properties in wide temperature range.
\end{abstract}

(c) 2013 Elsevier B.V. All rights reserved.

\section{Introduction}

In the present time there is a large number of papers (e.g. [1-5]) dedicated to investigation of the actinide metals and their compounds. This can be explained not only by their strategic importance, but also by their unusual physical properties. One of the least studied metals in this group is neptunium [6], which stands just in front of plutonium in the periodic table. The experimental data on specific heat is available only for $\alpha$-Np in the temperature range $0-300 \mathrm{~K}[7,8]$. At the same time $\mathrm{Np}$ possess a large value of the linear coefficient of electronic heat capacity $\left(14.2 \mathrm{~mJ} / \mathrm{K}^{2}\right)$, which is second after plutonium in the actinides series [1]. The estimation of coefficient of electronic heat capacity of Np based on the results of LDA-calculations of its electronic structure [9] gives the value that several times smaller than $14.2 \mathrm{~mJ} / \mathrm{K}^{2}$. It means that Np probably can be attributed to the group of strongly-correlated materials and thus can exhibit complicated temperature dependencies of the electronic contributions to the thermal and elastic properties.

On the other hand, it is known that the principal contribution to thermal and elastic properties is represented by the lattice subsystem. At the same time there is a number of indications to that $\mathrm{Np}$ can be characterized by strong anharmonicity of its lattice vibrations (the phonon anharmonicity), e.g. the enhanced values of its volumetric thermal expansion coefficient [10] and heat capacity $[7,8]$. The phonon anharmonicity of neptunium as well as its electronic subsystem can provide nonlinear in temperature contributions to the thermal and elastic properties (in particular, to the heat capacity).

\footnotetext{
* Corresponding author. Address: Ural Federal University, Physics Department, Mira Str. 19, 620002 Ekaterinburg, Russia. Tel./fax: +7 3433754538.

E-mail addresses: a.n.filanovich@ustu.ru (A. Filanovich), a.a.povzner@ustu.ru (A. Povzner).
}

In the papers [11-13] a self-consistent thermodynamic model has been developed, which on the basis of generalization of the Debye model, describes the effect of phonon anharmonicity on the thermal and elastic properties of metals. This model enabled to describe the unusual temperature dependencies of thermal properties of plutonium [13] and correctly determine the electronic contributions that in case of Pu are also affected by the spin anharmonicity [3,13].

In the present study we develop a self-consistent thermodynamic model for neptunium and make quantitative estimation of its phonon anharmonicity parameters. In terms of the developed model we has calculated the temperature dependencies of molar heat capacity, volumetric thermal expansion coefficient, bulk modulus and Debye temperature of $\alpha$-Np (exists in the range $0-554 \mathrm{~K}$ ) and $\beta$-Np (554-850 K). The $\gamma$-phase of neptunium has not been considered since it exists in a rather narrow temperature range prior to the melting point (850-913 K) and experimental data on the properties of this phase, necessary for the self-consistent calculations, are not available.

\section{Model description}

It is well established that in real solids the lattice vibrations are anharmonic and that leads to the effect of thermal expansion. The general form of potential of atoms interaction is defined by the series of terms of the form $a \xi^{\beta}$, where $\xi$ is the generalized displacement and $a, \beta$ are constants. In the harmonic approximation $\beta=2$ and therefore in the high temperature limit the energy per one mode equals $k T / 2$, whereas the total energy of crystal is $3 N k T$, where $N$ is the number of atoms, $k$ is Boltzmann's constant and $T$ is the temperature. In the presence of anharmonicity the sum over all modes will no longer equal $3 N k T$ and this can be taken into account via temperature-dependent characteristic Debye temperature. Exactly such an approach is implemented in the self-consistent thermodynamic model [11-13], in which the Debye 
temperature is no longer a constant and depends on the temperature and volume. The averaged Debye temperature is averaged value of the "partial" Debye temperatures $\theta_{l}$ and $\theta_{t}$ :

$\theta=\left(\frac{3}{1 / \theta_{l}^{3} 2 / \theta_{t}^{3}}\right)^{1 / 3}$.

The "longitudinal" Debye temperature corresponds to the longitudinal mode of acoustic vibrations

$\theta_{l}=\frac{h}{k_{B}}\left(\frac{6 \pi^{2} N_{A}}{V}\right)^{1 / 3} \sqrt{\frac{B+\frac{4}{3} G}{\rho}}$,

and the "transverse" Debye temperature corresponds to the transverse mode of acoustic vibrations

$\theta_{t}=\frac{h}{k_{B}}\left(\frac{6 \pi^{2} N_{A}}{V}\right)^{1 / 3} \sqrt{\frac{G}{\rho}}$,

where $\hbar, N_{A}$ are Plank and Avogadro constants, respectively; $\rho=\mu / V$ is mass density; $B$ and $G$ are bulk modulus and shear modulus, respectively, $\mu$ is the molar mass. The elastic modules that enters the expressions for Debye temperature components can be conventionally expressed through the bulk modulus and Poisson coefficient $\sigma$ :

$B+\frac{4}{3} G=\frac{1-\sigma}{1+\sigma} B=3 \Xi_{l} B ; \quad G=3 \frac{1 / 2-\sigma}{1+\sigma} B=3 \Xi_{t} B$.

In the expression (4) the following designations for the $\Xi(\sigma)$-functions are adopted

$\Xi_{l}=\frac{1-\sigma}{1+\sigma}, \Xi_{t}=\frac{1 / 2-\sigma}{1+\sigma}$.

With the said, the Debye temperature takes the following form:

$\theta=\frac{h}{k_{B}}\left(6 \pi^{2} N_{A}^{2}\right)^{1 / 3} \sqrt{\frac{3}{\mu}} \Xi^{1 / 2} B^{1 / 2} V^{1 / 6}$,

where the $\Xi(\sigma)$-function is

$\Xi=\left(\frac{3}{1 / \Xi_{l}^{3 / 2}+2 / \Xi_{t}^{3 / 2}}\right)^{2 / 3}$.

Thus we have replaced the dependence of Debye temperature on shear modulus by dependence on Poisson's coefficient $\sigma$, which is assumed to be temperature-independent and which in the present study is a fitting parameter of the model. Considering that bulk modulus $B$ is deduced from its thermodynamic definition (see below), we obtain the Eq. (6), which to some approximation can be applied to non-cubic metals in contrast to the initial Eqs. (2) and (3).

In order to calculate the "static" thermodynamic functions of crystal lattice of a paramagnetic metal one can use two thermodynamic potentials: the Gibbs thermodynamic potential (TDP) and the Helmholtz free energy (FE). The differentials of molar TDP and FE have the form:

$$
\begin{aligned}
& d \Phi=-S d T+V d P \\
& d F=-S d T-P d V .
\end{aligned}
$$

The derivatives of TDP and FE of different orders and with respect to different parameters enable to calculate the whole complex of the most important thermal and elastic properties. The expressions for TDP and FE can be written in the traditional additive form

$\Phi=\Phi_{0}+\Phi_{p h}+\Phi_{e}$.

Here $\Phi_{0}=\Phi_{0}(P)$ is the "constant" part of TDP, which is temperature independent but is a function of pressure; $\Phi_{p h}=\Phi_{p h}(\theta(P), T)$ is the molar lattice (phonon) part of TDP responsible for the contribution of acoustic vibrations of a solid:

$\Phi_{p h}=3 R\left(\frac{3}{8} \theta+T \phi(z)\right)$

where $z=\theta / T$ is the inverse reduced temperature and $\phi(z)=$ $\ln \left(1-e^{-z}\right)-D(z) / 3(D(z)$ is the standard tabulated Debye function). $\Phi_{e l}$ is the electronic part of TDP expressed as following:

$\Phi_{e l}=-\frac{\varsigma T^{2}}{2}$,

where $\varsigma$ is the coefficient of electronic heat capacity, which in the framework of the present study is temperature independent.

As one can see from (6) and (10) the Debye temperature $\theta$ enters the definition of the phonon part of thermodynamic potential $\Phi_{p h}=\Phi_{p h}(\theta, T)$ and at the same time is a function of temperature. Since all the calculated thermal and elastic properties are defined from the phonon TDP $\Phi_{p h}=\Phi_{p h}(\theta, T)$, the Debye temperature affects these properties. The change in the properties, in turn, leads to the Debye temperature renormalization. Thus one can construct an iterative procedure for the self-consistent calculations of temperature dependencies of the Debye temperature, thermal and elastic properties, that accounts for the effects of phonon anharmonicity. It is worth noting that thus accounted effects of the phonon anharmonicity are related to thermal expansion, whereas in general phonon anharmonicity can also manifest in peculiarities of the phonon spectrum, which are not captured by the present model. However, the features of phonon spectrum make significant impact primarily at low temperatures, while in a wide temperature range, which is the case for this study, the main effect is due to the thermal expansion.

In terms of the model under consideration we introduce the generalized dimensionless Gruneisen parameters, which for a thermodynamic quantity $f=f(T, V)$ at constant temperature and pressure are defined as

$\gamma_{f}=\frac{V}{f}\left(\frac{\partial f}{\partial V}\right)_{T P} ; \quad \gamma_{f}^{*}=\frac{V^{2}}{f}\left(\frac{\partial^{2} f}{\partial V^{2}}\right)_{T P}$.

It is clear that the generalized Gruneisen parameter $\gamma_{\theta}$ of the first order of the Debye temperature up to sign coincides with the well-known Gruneisen parameter of a solid $\Gamma=-\frac{\partial \ln \theta}{\partial \ln V}$, which characterizes the degree of lattice vibrations anharmonicity. As shown in [11] the Gruneisen parameters of the Debye temperature $\gamma_{\theta}$ and $\gamma_{\theta}^{*}$ in the framework of the self-consistent thermodynamic model are expressed through the Gruneisen parameters of bulk modulus $\gamma_{B}, \gamma_{B}^{*}$ and Poisson coefficient $\gamma_{\sigma}, \gamma_{\sigma}^{*}$. The parameters $\gamma_{B}$ and $\gamma_{B}^{*}$ depend on temperature and this leads to the temperature dependence of $\gamma_{\theta}$ and $\gamma_{\theta}^{*}$. The starting (at $T=0 \mathrm{~K}$ ) values of $\gamma_{B}$ and $\gamma_{B}^{*}$ as well as the values of $\gamma_{\sigma}, \gamma_{\sigma}^{*}, \gamma_{\sigma}, \gamma_{\varsigma}^{*}$ and Poisson coefficient $\sigma$ represent the parameters of the model.

Using the thermodynamic potential described by formulas (9)(11), we obtain the following expression for the molar heat capacity:

$$
\begin{aligned}
C(T)= & -T \frac{\partial^{2} \Phi}{\partial T^{2}} \\
& =3 R\left\{C_{V R}(z)\left[1-\frac{1}{z}\left(\frac{\partial \theta}{\partial T}\right)_{P}\right]^{2}-T\left[\frac{3}{8}+\frac{D(z)}{z}\right]\left(\frac{\partial^{2} \theta}{\partial T^{2}}\right)_{P}\right\}+\varsigma T,
\end{aligned}
$$

where $C_{V R}(z)$ is the standard Debye heat capacity, normalized to 3R. From the expression (13) one can see that in the absence of the temperature dependence of the Debye temperature, the molar heat capacity coincides with the Debye heat capacity $C_{V R}(z)$, i.e. with the heat capacity calculated within harmonic approximation. The self-consistent thermodynamic model enables to calculate the 
temperature dependence of Debye temperature in a self-consistent manner and thus account for an additional contribution to the molar heat capacity associated with the phonon anharmonicity.

The molar volume is defined by the expression

$V(T)=\left(\frac{\partial \Phi}{\partial P}\right)_{T}=V_{0}-\frac{3 R \theta \gamma_{\theta}}{B}\left(\frac{3}{8}+\frac{D(z)}{z}\right)+\frac{\varsigma \gamma_{\varsigma} T^{2}}{2 B}$,

where $V_{0}$ is the volume at $T=0 \mathrm{~K}$, the second term is the lattice contribution and the third term is due to the electronic subsystem. Then for the volumetric thermal expansion coefficient (VCTE) one can derive the formula

$$
\begin{aligned}
O(T)= & \frac{1}{V}\left(\frac{\partial V}{\partial T}\right)_{P}=-\frac{3 R \theta \gamma_{\theta}}{V B}\left\{C_{V R}(z)\left[1-\frac{T}{\theta}\left(\frac{\partial \theta}{\partial T}\right)_{P}\right] \frac{1}{\theta}+\left[\frac{3}{8}+\frac{D(z)}{z}\right]\left[\frac{1}{\theta}\left(\frac{\partial \theta}{\partial T}\right)_{P}\right.\right. \\
& \left.\left.+\frac{1}{\gamma_{\theta}}\left(\frac{\partial \gamma_{\theta}}{\partial T}\right)_{P}-\frac{1}{B}\left(\frac{\partial B}{\partial T}\right)_{P}\right]\right\}+\frac{\zeta \gamma_{S}}{V B} T .
\end{aligned}
$$

Finally, for the bulk modulus we obtain the expression

$$
\begin{aligned}
B(T)= & V\left(\frac{\partial^{2} F}{\partial V^{2}}\right)_{T} \\
& =B_{0}+\frac{3 R}{V}\left\{\frac{3}{8} \gamma_{\theta}^{*} \theta-T\left[\gamma_{\theta}^{*} C_{V R}(\theta / T)-\gamma_{\theta}^{*} D(\theta / T)\right]\right\}-\frac{1}{2 V} \gamma \zeta^{*} \zeta T^{2},
\end{aligned}
$$

where $B_{0}$ is the bulk modulus at $T=0 \mathrm{~K}$. We should note that the values of $V_{0}$ and $B_{0}$ also belong to the list of fitting parameters, i.e. are estimated from the condition of the best agreement between the experimental and calculated data. Thus the present model contains a rather big amount of fitting parameters, but all these parameters have clear physical sense. Furthermore, the calculations of the properties are performed in terms of physically justified model, which compares favorably with the calculations based on empirical relationships that operate with some abstract polynomial dependencies.

\section{The results and discussion}

By implementing the developed model we have performed the calculations of thermal and elastic properties of $\alpha$ - and $\beta$-phases of neptunium. For the calculations of electronic contributions to the properties of $\alpha$ - and $\beta$-phases of $\mathrm{Np}$ we used the value $14.2 \mathrm{~mJ} /$ $\mathrm{K}^{2}$ for the electronic heat capacity coefficient, which was obtained in [7] from fitting the low-temperature $(\alpha-\mathrm{Np})$ experimental data on molar heat capacity by a conventional sum of cubic and linear terms. In the case of $\beta$-Np the electronic heat capacity coefficient is probably different, but keeping in mind that there is no any kind of experimental data for heat capacity of $\beta-\mathrm{Np}$, to a first approximation one can adopt the value $14.2 \mathrm{~mJ} / \mathrm{K}^{2}$ for calculating the properties of both phases considered. As already mentioned above for neptunium there is rather limited amount of the available experimental data, but the availability of the data [10] on VCTE and mass density enabled us to make an estimation of the parameters, characterizing the phonon anharmonicity of $\alpha$ - and $\beta$-phases of $\mathrm{Np}$ and perform the self-consistent calculations of the thermal end elastic properties. The obtained values for the parameters are listed in Table 1.

It should be noted that the Poisson coefficient $\sigma$, which strongly affects the Debye temperature and (as a consequence) all thermal and elastic properties, can be determined from either data on elastic modules [5] or on the mean-square displacement (MSD) of atoms from the equilibrium positions [12]. However, in the case of Np experimental data neither on elastic modules nor on MSD is available. Therefore in the present study the Poisson ratio along with the other parameters listed in Table 1 has been found from the condition of the best agreement between the experimental and calculated temperature dependencies of neptunium properties. In evaluating the Poisson's ratio of $\alpha$ - and $\beta$-phases of $\mathrm{Np}$
Table 1

Lattice and electronic parameters of $\alpha$ - and $\beta-\mathrm{Np}$.

\begin{tabular}{lrr}
\hline & $\alpha-\mathrm{Np}$ & \multicolumn{1}{c}{$\beta-\mathrm{Np}$} \\
\hline$\gamma_{B 0}$ & -0.20 & 0.40 \\
$\gamma_{B 0}^{*}$ & 1.96 & 1.48 \\
$\sigma$ & 0.33 & 0.40 \\
$\gamma_{\sigma}$ & 3.18 & 1.24 \\
$\gamma_{\sigma}^{*}$ & 4.08 & -3.56 \\
$\gamma_{\zeta}$ & 4.00 & 2.00 \\
\hline
\end{tabular}

we proceeded from the fact that at the phase transition from $\alpha$ to $\beta$-phase in plutonium, which is similar to the phase transition from $\alpha$ - to $\beta$-phase in $\mathrm{Np}$, an essential increase in $\sigma$ is observed ( $\sigma=0.18$ in the case of $\alpha$-Pu while $\sigma=0.28$ in the case of $\beta-\mathrm{Pu}$ ). Thus for $\alpha$-Np we obtained $\sigma=0.33$, whereas for $\beta$-Np $\sigma=0.40$.

Since according to [10] the phase transition from $\alpha$ to $\beta$ phase occurs in the temperature range from 538 to $558 \mathrm{~K}$, in all the figures we plot the data for both phases considered in this temperature range. Fig. 1 presents the results of calculations of molar isobaric heat capacity $\left(C_{p}\right)$ of neptunium, which shows that our model explains the experimental data that is available up to $300 \mathrm{~K}$. The values obtained for the $\alpha$-phase at higher temperatures as well as the values for the $\beta$-phase in the entire temperature range of its existence have predictive character and point to the necessity of further experimental and theoretical investigations of neptunium heat capacity. Also in Fig. 1 we show the heat capacity $C_{v}$ of $\alpha$-Np calculated at constant volume, i.e. neglecting the anharmonic effects. One can see that at temperatures higher than Debye temperature the $C_{v}$ curve is essentially lower than the $C_{p}$ curve, reflecting the sufficient impact of the phonon anharmonicity on the heat capacity of neptunium.

Fig. 2 shows the calculated temperature dependencies of the volumetric thermal expansion coefficient of neptunium in comparison with the experimental data [10]. Both $\alpha$ - and $\beta-N p$ exhibit VCTE values, which are record high in comparison with other metals, e.g. transition metals palladium and platinum [12], and which are comparable to alpha-plutonium [14]. As can be seen from Fig. 2, although there is a finite electronic contribution to the VCTE of Np, the anomalously large values of VCTE are mainly caused by the lattice subsystem, particularly by the phonon anharmonicity.

In Fig. 3 we demonstrate our results of the bulk modulus calculation of neptunium. According to our data at the point of phase transition from $\alpha$ - to $\beta$-phase of $\mathrm{Np}$ a quite dramatic change of the bulk modulus occurs: $\beta$-Np exhibits lower values of the bulk

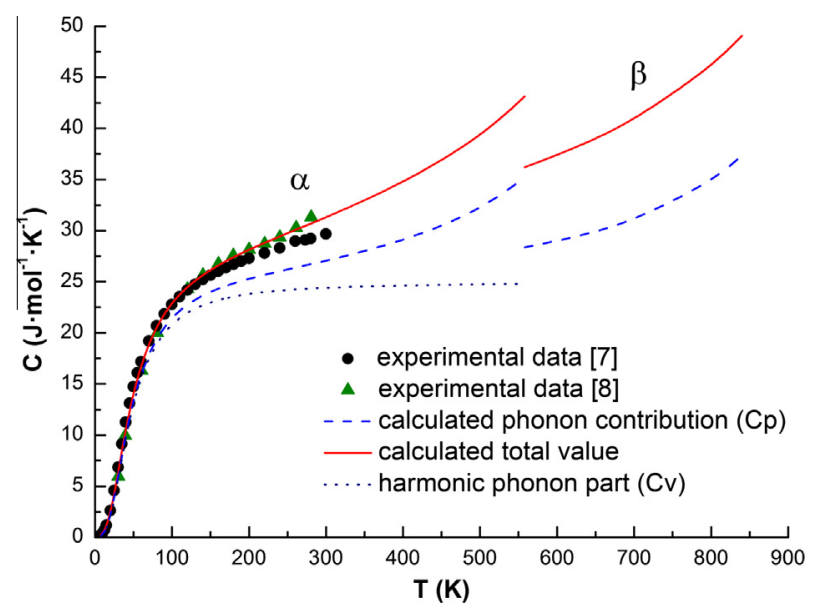

Fig. 1. Temperature dependence of the molar heat capacity of neptunium 


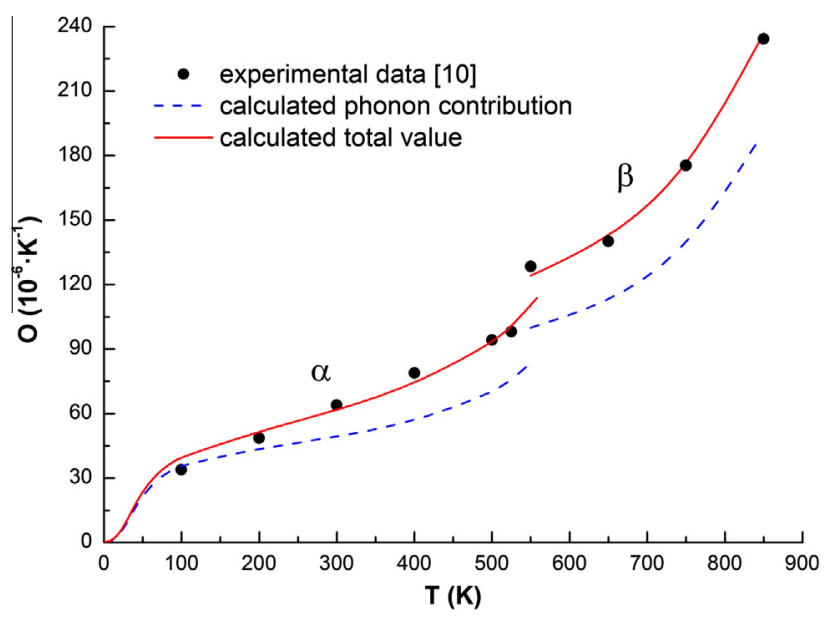

Fig. 2. Temperature dependence of the volumetric thermal expansion coefficient of neptunium.

modulus and its weaker temperature dependence. It is worth noting that the similar changes are observed at the phase transition from $\alpha$ - to $\beta$-phase of plutonium [5]. However, in order to verify the results of our calculations, a big amount of experimental data on the bulk modulus of neptunium is required, whereas at the present moment this data is extremely sparse. The Debye temperature of neptunium at the phase transition from $\alpha$ - to $\beta$-phase undergoes the changes, which are similar to those of the bulk modulus (Fig. 4). From Fig. 4 one can see that, in general, our data on the Debye temperature of $\alpha-\mathrm{Np}$ are in agreement with the data [7] obtained from the analysis of experimental data on heat capacity of $\alpha$-Np.

As clear from Fig. 5, where the temperature dependencies of neptunium mass density defined as $\rho=\mu / V$ are shown, the developed model enables to describe the experimental data in a wide temperature range excluding some discrepancies observed in the vicinities of the phase transitions. The discrepancies can be explained by the fact that the experimental data shown in Fig. 5 was obtained not by direct density measurements but has been converted from the data [10] on the relative elongation of the sample.

Fig. 6 shows the results of calculations of the Gruneisen parameter $\Gamma=-\frac{\partial \ln \theta}{\partial \ln V}$ of $\alpha$ - and $\beta$-Np as a function of temperature. This parameter describes the volume dependence of Debye temperature and, in more general sense, provides information on the

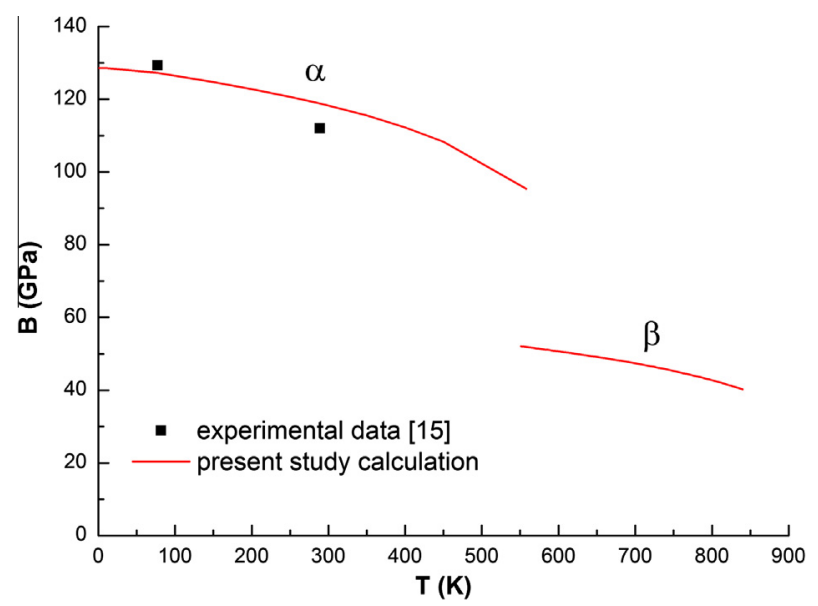

Fig. 3. Temperature dependence of the bulk modulus of neptunium. (See abovementioned references for further information.)

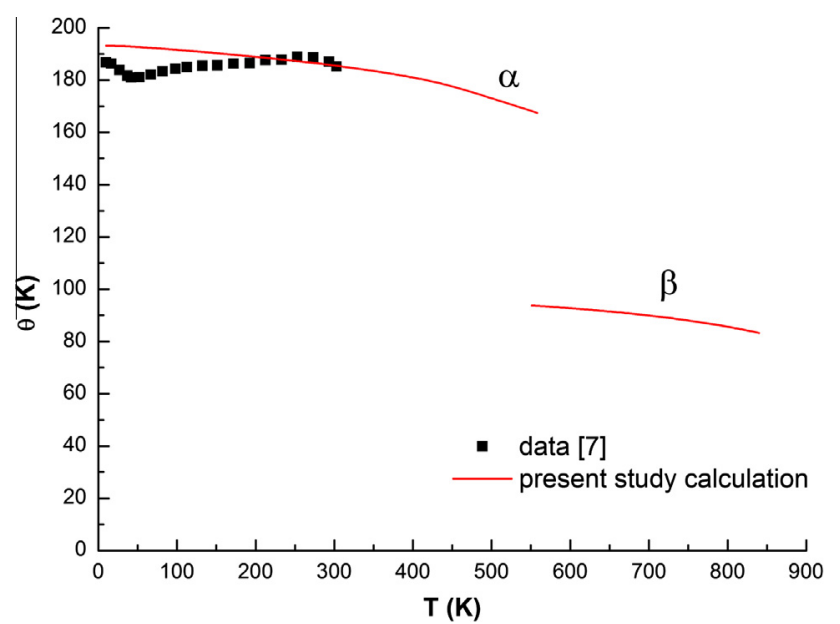

Fig. 4. The Debye temperature of neptunium.

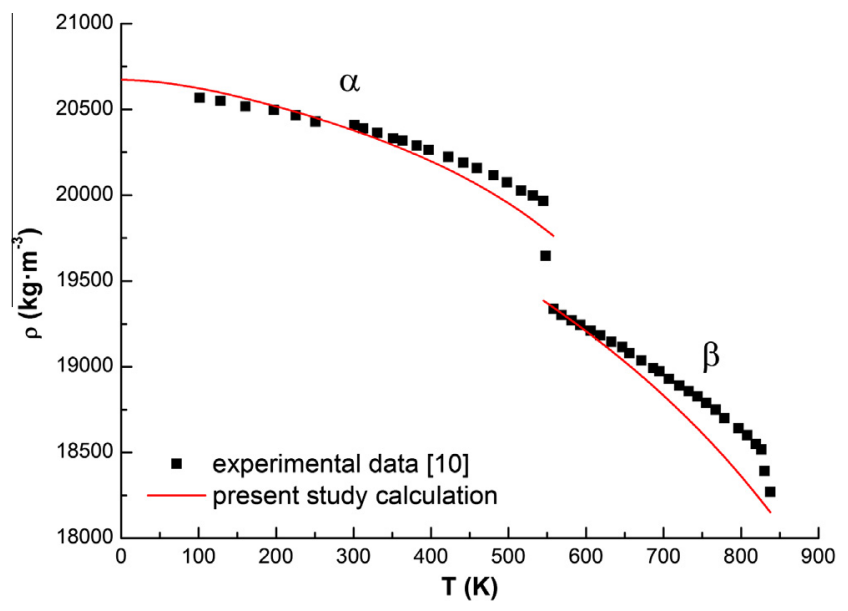

Fig. 5. Temperature dependence of the mass density of neptunium.

volume dependence of dispersion curves of phonon spectrum $\omega(q)$, i.e. enables to define the degree of the lattice (phonon) anharmonicity. From Fig. 6 one can see that with growing temperature the Gruneisen parameter of $\alpha$-Np exhibits sufficiently rapid increase, however, exactly at the point of the phase transition a dramatic drop of the phonon anharmonicity occurs followed by

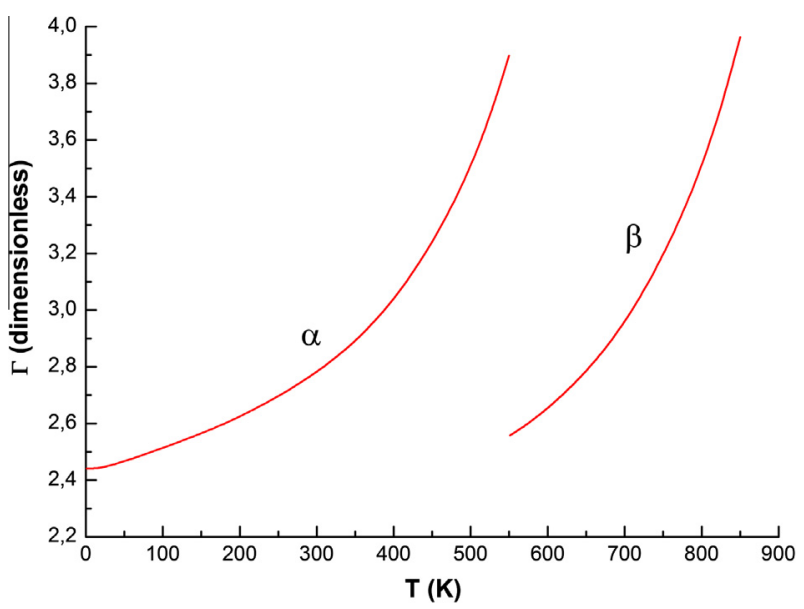

Fig. 6. Temperature dependence of the Gruneisen parameter of neptunium (results of the present study calculations). 
its increase in the $\beta$-phase. The values of the Gruneisen parameter, which were reached at the point of the phase transition from $\alpha$ - to phase are achieved again only in the temperature range close to the transition point to the $\gamma$-phase of neptunium. As in the case of VCTE neptunium demonstrates the values of Gruneisen parameter that are much higher as compared to transition metals. Thus, for palladium and platinum in the entire range of their existence $\Gamma$ does not exceed 3.1 [12]. Comparing to plutonium [5] we can point that neptunium possess the values of the Gruneisen parameter, which are typical for $\delta$-Pu $(\Gamma=3.8$ at $T=480 \mathrm{~K})$ but higher than in the case of $\beta-\mathrm{Pu}(\Gamma=2.3$ at $T=450 \mathrm{~K})$ and $\gamma-\mathrm{Pu}(\Gamma=1.9$ at $T=550 \mathrm{~K})$. This confirms the presence of strong phonon anharmonicity of neptunium.

\section{Conclusions}

In the present study in terms of the self-consistent thermodynamic model we have calculated the temperature dependencies of thermal end elastic properties of $\alpha$ - and $\beta$-phases of neptunium. Many of the obtained dependencies are predictive and point to the necessity of further experimental and theoretical investigations of neptunium, especially its $\beta$-phase. We can summarize that a significant role in formation of the features of neptunium thermal and elastic properties belongs to the phonon anharmonicity.

It is established that the value $14.2 \mathrm{~mJ} / \mathrm{K}^{2}$ for the electronic heat capacity coefficient adopted from [7] enables to obtain a reason- able agreement with the experimental data. However, refinement and understanding of this value as well of the electronic properties of neptunium in general, presents the subject for further studies.

\section{Acknowledgements}

The study was supported by The Ministry of education and science of Russian Federation, Project 14.A18.21.0737.

\section{References}

[1] K.T. Moore, Rev. Mod. Phys. 81 (2009) 235

[2] M. Boivineau, J. Nucl. Mater. 392 (2009) 568.

[3] A. Solontsov, V.P. Antropov, Phys. Rev. B. 81 (2010) 214402.

[4] A. Kutepov, K. Haule, S.Y. Savrasov, G. Kotliar, Phys. Rev. B. 85 (2012) 155129.

[5] Y. Suzuki, V.R. Fanelli, J.B. Betts, F.J. Freibert, C.H. Mielke, J.N. Mitchell, M. Ramos, T.A. Saleh, A. Migliori, Phys. Rev. B. 84 (2011) 064105.

[6] J. Ibers, Nat. Chem. 996 (2010), http://dx.doi.org/10.1038/nchem.888.

[7] J.A. Lee, K. Mendelssohn, P.W. Sutcliffe, Proc. R. Soc. Lond. A. 317 (1987) 303.

[8] T.A. Sandenaw, J. Phys. Chem. Solids 26 (1965) 1075.

[9] R.C. Albers, A.M. Boring, J.M. Wills, L.E. Cox, O.E. Eriksson, N.E. Christensen, Phys. Rev. B. 54 (1996) 14405.

[10] B. Cort, J. Less Common Met. 135 (1987) L13-L17.

[11] V.Y. Bodryakov, A.A. Povzner, I.V. Safonov, Tech. Phys. 51 (2006) 216.

[12] A.A. Povzner, A.N. Filanovich, High Temp. 49 (2011) 674.

[13] A.A. Povzner, A.G. Volkov, A.N. Filanovich, Phys. Solid State 53 (2011) 1761.

[14] A.E. Kay, R.G. Loasby, Philos. Magn. 9 (1964) 37.

[15] V.E. Zinoviev, Thermophysical Properties of Metals at High Temperatures: A Handbook, Metallurgiya, Moscow, 1989. 